\title{
Near-wall modelling and free-stream turbulence effects on square cylinder unsteady heat transfer
}

\author{
X. Chen and H. Xia \\ Department of Aeronautical and Automotive Engineering \\ Loughborough University, Loughborough, Leicestershire, LE11 3TU, UK, x.chen@lboro.ac.uk
}

\begin{abstract}
Unsteady flow passing a heated square cylinder has been investigated using a hybrid LES-RANS approach at a moderate Reynolds number of 22,050. Two near-wall RANS models are blended smoothly to the LES region. The two models applied have successfully reproduced time- and phase-averaged flow field. Encouraging convective heat transfer has been predicted. An increase in the convective heat flux is found on the cylinder top/bottom surface with imposed free-stream turbulence. More accurate prediction of velocity and Nusselt number profiles has been made by the use of LES- $k \omega$ model.
\end{abstract}

\section{Introduction}

Unsteady flows are common in many industrial configurations such as flows around compressors and turbines. In such cases, turbulence plays an important role in solid surface temperature distribution as a result of convective heat transfer. For example, hot gases from the combustion chamber hit turbine blades while cooling flows are injected from the blades' cavity. Reproducing the surface temperature distribution is essential for both design and optimization purposes. However, flow separation and reattachment, mixing of the cold \& hot streams and wake vortices greatly affect the surface temperature distribution. Thus, it is of great importance to have accurate prediction of these unsteady flows that relate to surface heat transfer.

Flow passing a heated square cylinder is widely used as a classical case in studies of unsteady flow and heat transfer. The separation, flow recirculation and vortex shedding are keys to the convective heat transfer on cylinder surfaces. Bosch and Rodi (1998) studied this case using unsteady Reynolds-averaged Navier-Stokes (URANS) (widely used in industrial applications). Although some main parameteres like lift/drag coefficient and Strouhal number were predicted, the URANS was unable to reproduce details of turbulent structures, especially those near the cylinder surfaces that were essential to heat transfer. Therefore, as high-performance computing resources becoming more affordable, researchers moved to large-eddy simulation (LES) and detached-eddy simulation (DES) for more accurate prediction of turbulent flows. Rodi et al. (1997), Barone \& Roy (2006) and Wiesche (2007) conducted LES/DES studies on structured grids. However, it is not possible to use structured grid for most industrial applications and unstructured grid is the preferred choice. As the numerical approach of unstructured grid is different from that of the structured grid, results of the LES/DES simulations on structured grid may not be sufficient and validation on unstructured grid is needed. More recently, Boileau et al. (2013) showed that wall resolved LES on unstructured grid provided good prediction on unsteady heat transfer using sufficiently high grid resolution and small time-step size, which could lead to a high demand in computing resources.

In the present work, flow passing a heated square cylinder is investigated numerically using a hybrid LES-RANS approach with unstructured grids. The hybrid LES-RANS model used here attempts to combine the advantages of LES in resolving vortices away from the wall with welldeveloped RANS for near-wall treatment. Two RANS models are used: the Spalart-Allmaras 
(SA) model and SST $k-\omega$ model, and blended into the LES zone smoothly. Free-stream turbulence is imposed to the inflow. Validation of the numerical results is conducted by comparing the flow field near the cylinder top surface and in the wake as well as surface heat transfer profiles with experiments.

\section{Numerical method}

The Favre-average compressible Navier-Stokes equations for ideal gas are solved in conservation form,

$$
\frac{\partial \overline{\mathbf{Q}}}{\partial t}+\frac{\partial \overline{\mathbf{F}}_{i}^{i n v}}{\partial x_{i}}-\frac{\partial \overline{\mathbf{F}}_{i}^{v i s}}{\partial x_{i}}=0
$$

The conservative variables are $\overline{\mathbf{Q}}=\left[\bar{\rho}, \bar{\rho} \widetilde{u}_{i}, \widetilde{E}\right]^{T}$, the inviscid and viscid fluxes are defined as $\overline{\mathbf{F}}_{i}^{i n v}=\widetilde{u}_{i} \overline{\mathbf{Q}}+\left[0, \delta_{1 i} \bar{p}, \delta_{2 i} \bar{p}, \delta_{3 i} \bar{p}, \widetilde{u}_{i} \bar{p}\right]^{T}$ and $\overline{\mathbf{F}}_{i}^{v i s}=\left[0, \widetilde{\tau}_{1 i}, \widetilde{\tau}_{2 i}, \widetilde{\tau}_{3 i}, \widetilde{\tau}_{k i} \widetilde{u}_{k}+\widetilde{q}_{i}\right]^{T}$. The stress tensor $\widetilde{\tau}_{i j}$, total energy $\widetilde{E}$ and heat flux $\widetilde{q}_{j}$ satisfies,

$$
\widetilde{\tau}_{i j}=2\left(\mu+\mu_{T}\right)\left(\widetilde{S}_{i j}-\frac{1}{3} \partial_{j} \widetilde{u}_{j} \delta_{i j}\right), \quad \widetilde{E}=\bar{\rho} \widetilde{e}+\frac{1}{2} \bar{\rho} \widetilde{u}_{i} \widetilde{u}_{i}, \quad \widetilde{q}_{i}=-\left(k+k_{T}\right) \frac{\partial \widetilde{T}}{\partial x_{i}}
$$

where the turbulent thermal conductivity is computed from $k_{T}=\mu_{T} C p / \operatorname{Pr}$. An extra equation of state $\bar{p}=\bar{\rho} R \widetilde{T}$ is also included to define the relation between pressure, density and temperature for ideal gas.

\subsection{Near-wall modelling}

The standard SA model is used for the near-wall RANS. The turbulent eddy viscosity is defined as $\mu_{T}=\rho \widetilde{\nu} f_{\nu 1}$, where $\widetilde{\nu}$ satisfies the transport equation,

$$
\frac{\partial \widetilde{\nu}}{\partial t}+\widetilde{u}_{i} \frac{\partial \widetilde{\nu}}{\partial x_{i}}=c_{b 1} \widetilde{S} \widetilde{\nu}-c_{w 1} f_{w}\left(\frac{\widetilde{\nu}}{d}\right)^{2}+\frac{1}{\delta}\left[\nabla \cdot((\nu+\widetilde{\nu}) \nabla \widetilde{\nu})+c_{b 2}(\nabla \widetilde{\nu})^{2}\right]
$$

The SST $k-\omega$ model is also applied for the near-wall modelling. It solves two transport equations to determine the turbulent kinetic energy and dissipation,

$$
\begin{gathered}
\frac{\partial \rho k}{\partial t}+\frac{\partial \rho u_{i} k}{\partial x_{i}}=P-\beta^{*} \rho \omega k+\frac{\partial}{\partial x_{i}}\left[\left(\mu+\delta_{k} \mu_{T}\right) \frac{\partial k}{\partial x_{i}}\right] \\
\frac{\partial \rho \omega}{\partial t}+\frac{\partial \rho u_{i} \omega}{\partial x_{i}}=\left(\frac{\gamma}{\nu_{t}}\right) P-\beta \rho \omega^{2}+\frac{\partial}{\partial x_{i}}\left[\left(\mu+\delta_{\omega} \mu_{T}\right) \frac{\partial \omega}{\partial x_{i}}\right]+2\left(1-F_{1}\right) \frac{\rho \delta_{\omega_{2}}}{\omega} \frac{\partial k}{\partial x_{i}} \frac{\partial \omega}{\partial x_{i}}
\end{gathered}
$$

where the turbulent eddy viscosity is defined as,

$$
\mu_{T}=\frac{\rho a_{1} k}{\max \left(a_{1} \omega, \Omega F_{2}\right)}
$$

The SST $k-\omega$ model is well-known for combining the accuracy of $k-\omega$ model in resolving the near wall boundary layer and the low free-stream sensitivity of the $k-\varepsilon$ like model (Menter et al. 2003). It is therefore expected that the SST $k-\omega$ model would give a better prediction of the flow field near the top/bottom and rear surfaces of the square cylinder. 


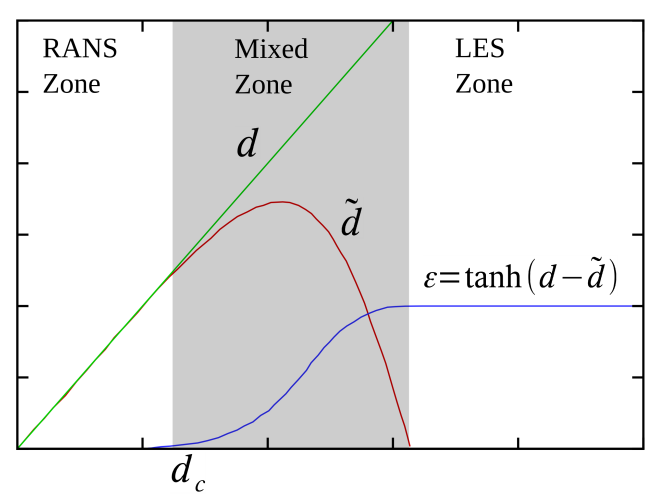

Figure 1: Hybrid LES-RANS approach

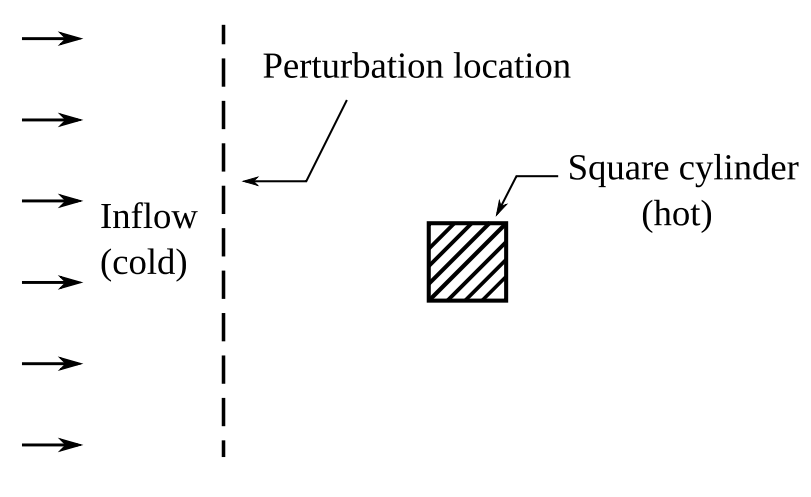

Figure 2: Schematic of the flow domain

\subsection{LES-RANS Hybridization}

The RANS and LES blending is achieved by using a Hamilton-Jacobi equation,

$$
|\nabla \widetilde{d}|=1+f(\widetilde{d}) \nabla^{2} \widetilde{d}+g(\widetilde{d}), \quad f(\widetilde{d})=\varepsilon_{0} \tilde{d}, \quad g(\widetilde{d})=\varepsilon_{1}\left(\frac{\widetilde{d}}{d_{c}}\right)^{2}
$$

where $\tilde{d}$ is the modified wall distance, $d_{c}$ is the RANS cut-off distance typically at $y^{+}=60, f$ and $g$ are the control functions. As Figure 1 shows, the RANS and LES regions are defined by the nearest wall distance $d$. The blending of RANS and LES in the mixed zone is controlled by $\tilde{d}$ and $\varepsilon$ ranges from 0 to 1 corresponding to the weighting of LES. A smooth transition in eddy viscosity field is therefore obtained and helps maintain good numerical stability. $\varepsilon=0$ means no explicit SGS model is used, which is often referred to as implicit or numerical LES. This hybrid LES-RANS approach has been successfully applied to quite a few other studies (e.g. Mitchell et al. 2006, Xia \& Tucker 2012)

\subsection{Spatial and temporal discretization}

Viscous terms are discretized using central differencing while a MUSCL type scheme is used for inviscid fluxes at the common face of two neighbour cells,

$$
\mathbf{F}=\frac{1}{2}\left(\mathbf{F}_{L}+\mathbf{F}_{R}\right)-\sigma \frac{1}{2}\left|\frac{\partial \mathbf{F}}{\partial \mathbf{Q}}\right|\left(\mathbf{Q}_{R}-\mathbf{Q}_{L}\right)
$$

The up-winding term is controlled by parameter $\sigma \in[0.1,1] . \mathbf{Q}$ and $\mathbf{F}$ are the conservative and flux vectors. Subscripts $L$ and $R$ represent the immediate left and right position of the common flux face, where piecewise linear reconstructions are performed from cell centres to give a second-order spatial accuracy. Moreover, the dual-time advancing is employed with the outer physical time discretized by a three-level backward Euler scheme, thus leading to a second-order temporal accuracy. The inner pseudo time is advanced by a three-stage RungeKutta scheme. As the outer time is discretized implicitly, it allows larger physical time steps to increase the efficiency compared with explicit time marching (Xia 2005).

\subsection{Free-stream turbulence generation}

Free-stream turbulence (FST) is imposed to the upstream of the cylinder by perturbing the velocity components. This perturbation in velocity field is expected to naturally develop into realistic 


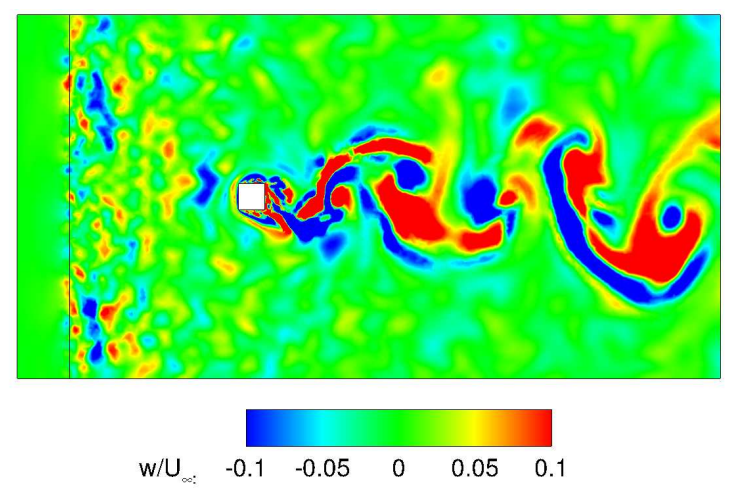

Figure 3: A slice of the instantaneous $z$ velocity contours with FST

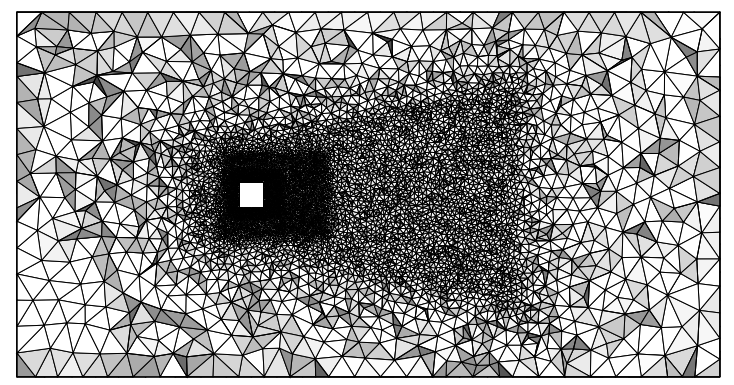

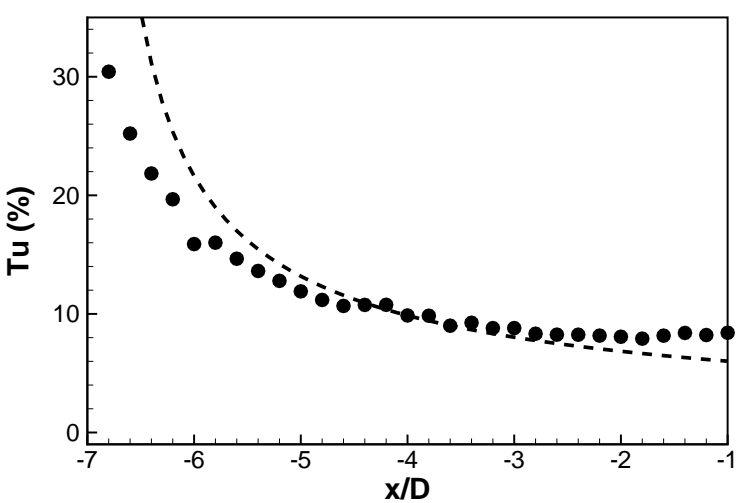

Figure 4: Decay of turbulence intensity at $y=4 D: \bullet$, Simulation; - -, Analytical

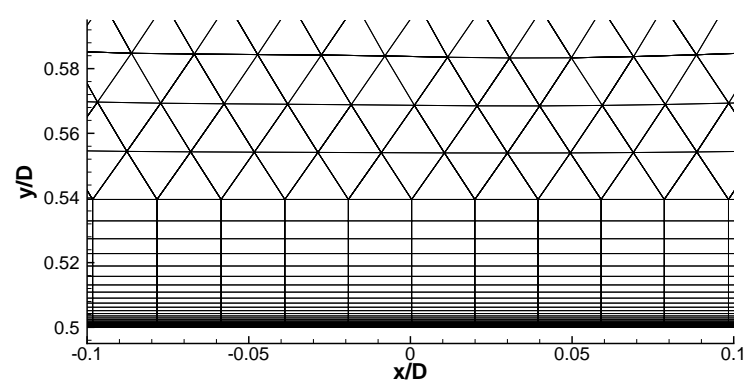

Figure 5: Cut-away view of the unstructured mesh (left) and enlarged view of the prism layers (right)

turbulence in the downstream region. This technique, although simple, was found quite effective by Pakora et al. (2011) in the near-wall region of a nozzle flow. Figure 2 shows a schematic of the perturbation location in the flow domain. The velocity perturbation is generated by,

$$
u^{\prime}=I U_{\infty} \theta, \quad \theta=\sqrt{-2 \ln \left(k_{1}\right)} \cos \left(2 \pi k_{2}\right), \quad k_{1}, k_{2} \in(0,1]
$$

where $U_{\infty}$ is the free-stream velocity, $I$ is a control coefficient, $k_{1}$ and $k_{2}$ are two random variables and $\theta$ is a random variable following a standard Gaussian distribution. More details of the random number generation can be found in Box \& Muller (1958). In the present study, the perturbation plane is set to be at $x=-7 D$ upstream of the cylinder. $1 / 2$ and $1 / 4$ of the turbulent velocity are applied respectively to the neighbouring and next level cells of the perturbation plane (i.e. $\mathrm{I} \pm 1 \& \mathrm{I} \pm 2$ ). This disturbed velocity field is interpolated trilinearly to the unstructured cells. Figure 3 illustrates the imposed FST and its development downstream by the $z$-velocity contours. The turbulence intensity of the simulated FST at $y=4 D$ away from the cylinder is presented and compared to the analytical solution of Baines \& Peterson (1951) in Figure 4.

\section{Configurations}

\subsection{Computational setup}

The square cylinder case has been studied by various experiments and simulations. In the present study, the diameter of the cylinder is $D=1 \mathrm{~cm}$. The flow domain is $14 D$ in height, $27 \mathrm{D}$ in length and $4 \mathrm{D}$ in span, which maintains the same blockage ratio as the experiment of Lyn \& Rodi (1994). 


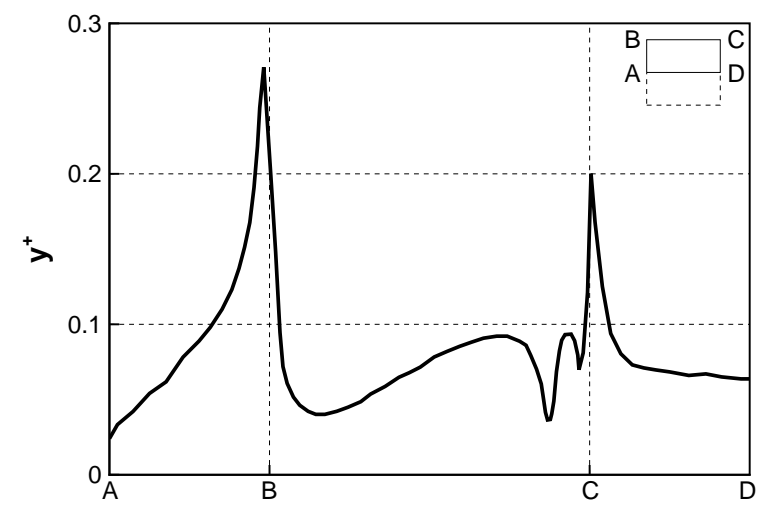

Figure 6: Time-averaged $y^{+}$profile for first boundary layer on cylinder surfaces

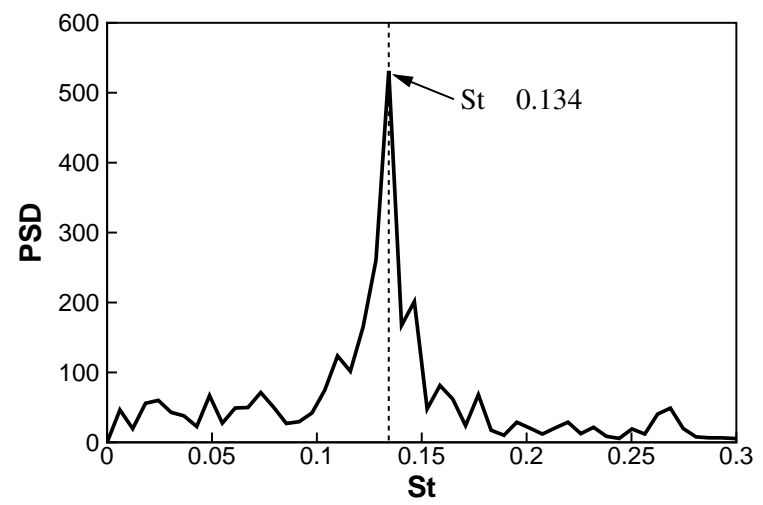

Figure 7: FFT of the raw pressure signal at centre line of cylinder top surface

The incoming air is under the standard atmosphere pressure at 101,325 $\mathrm{Pa}$. The temperature of the inflow is $300 \mathrm{~K}$, thus resulting a inlet Mach number of 0.108 . The top, bottom and side surfaces of the flow domain are treated as slip walls, while a no-slip and isothermal boundary condition at $330 \mathrm{~K}$ is applied to the cylinder surfaces. Change of flow temperature in the boundary layer due to the temperature difference between the wall and the free-stream induces a variation of fluid properties. Therefore, the Reynolds number is calculated based on a film temperature $T_{f}=\frac{1}{2}\left(T_{\infty}+T_{\text {wall }}\right)$, which results in $R e=22,050$.

A hybrid mesh of 4 million cells has been generated for numerical simulations. The cylinder corner is slightly rounded to allow better quality of the prism layers. As Figure 5 illustrated, two refinement regions in the wake and close to the cylinder can be observed to help resolving the flow recirculation near the wall and vortices in the wake. The cell height of the first prism layer $\Delta h$ is set to $10^{-4} D$, which limits the time-averaged $y^{+}$around the cylinder to be under 0.3 as Figure 6 shows. This shows that the first prism layer is small enough for the near-wall models to resolve the effect of the wall without the use of a wall function.

\subsection{Data processing method}

Lyn and Rodi (1994) and Lyn et al. (1995) investigated the velocity field near the top surface and in the wake using laser Doppler velocimetry. Time-averaged and phase-averaged velocities and fluctuations are provided and can be used to evaluate how our method captures the flapping shear layer, reversed flows and vortices in the wake. Durão et al. (1988) also provided some velocity data at $R e=14,000$ with a free-stream turbulence intensity of about $6 \%$. Another velocity data set measured using phase Doppler anemometry by Fohanno \& Martinuzzi (2004) is also included. In terms of pressure coefficient on cylinder surfaces, experimental profiles are available from Bearman \& Obasaju (1982) at $R e=20,000$ and Igarashi (1985) at $R e=$ 37, 000. For the von Kármán instability, Lyn et al. (1995), Durão et al. (1988) and Bearman \& Obasaju (1982) provided the Strouhal number as a reference of the vortex shedding frequency. This frequency is obtained by applying a fast Fourier transform (FFT) to the pressure signal taken from the centre line of the cylinder top surface. More detailed validation is performed by comparing the phase-averaged velocities above the cylinder top surface and in the near wake with results from Lyn et al. (1995).

Considering the validation of heat transfer results, Igarashi (1985) measured global and local time-averaged Nusselt number on cylinder surfaces at different Reynolds numbers. A correla- 
tion is also provided by Igarashi (1985) for the global averaged Nusselt number,

$$
\overline{N u}_{g}=0.14 R e^{0.66}
$$

which is valid for $5,000 \leqslant R e \leqslant 60,000$. As none of Igarashi's experiments are at the present Reynolds number ( $R e=22,050)$, Igarashi's correlation is transformed for scaling the Nusselt number profile at the closest Reynolds number $(R e=18,500)$,

$$
\overline{N u}_{s}=\overline{N u}_{\exp }\left(\frac{R e}{R e_{e x p}}\right)^{0.66}
$$

Yoo et al. (2003) measured the mass transfer of flow passing a square cylinder with the naphthalene-sublimation technique. The local Sherwood number profiles on cylinder surfaces can be used to derive the local Nusselt number profile using a analogy between heat and mass transfer. This analogy is expressed as,

$$
N u=S h\left(\frac{P r}{S c}\right)^{1 / 3}
$$

where the Prandtl number of air is $\operatorname{Pr}=0.71$ and the Schmidt number of naphthalene $S c=$ 2.53, obtained from the correlation of Cho et al. (1992) taken at temperature of $303 \mathrm{~K}$, the same as Yoo et al.'s experiment.

The instantaneous flow variable, $f(t)$, can be decomposed into a time-averaged component and a 'random' component, or a phase-averaged component and a 'random' component,

$$
f(t)=\bar{f}+f^{\prime}=\langle f\rangle+\langle f\rangle^{\prime}
$$

where $\bar{f}$ and $\langle f\rangle$ are the time- and phase-averaged parameters, $f^{\prime}$ and $\langle f\rangle^{\prime}$ are the fluctuation components. Root-mean-square value of the fluctuation part is introduced for the validation of these 'random' components. The phase-averaged parameter is obtained by averaging the instantaneous variable of all the time-steps that have the same phase angle $(\varphi)$ in vortex shedding cycles. Following Lyn \& Rodi (1994), the phase angles are defined on an instantaneous spanwise-averaged pressure signal $p(t)$ taken from the centre line of the cylinder top surface. This raw pressure signal is then processed by a low pass filter, whose cutting frequency equals to the vortex shedding frequency derived from FFT as Figure 7 shows. The peaks and valleys of the filtered signal are used to define every half of the vortex shedding period, where 10 separate phase angles are selected. Figure 8 shows an example of the phase angles on the filtered pressure signal.

\section{Results and discussion}

\subsection{Time-averaged results}

The time-averaged pressure coefficient profiles are presented in Figure 9. Good agreement can be seen between the numerical results and the experimental data from Igarashi (1985) $\bullet$ ) and Bearman \& Obasaju (1982) ( $\nabla$ ). The value of time-averaged pressure coefficient of the LES- $k \omega$ model on top/bottom surface reduces from corner $\mathrm{B}$ to corner $\mathrm{C}$, which is also shown in profiles of experiments. This reveals that the speed of the reversed flow reduces when moving upstream from corner $\mathrm{C}$ to corner $\mathrm{B}$.

Figure 10 illustrates the time-averaged $x$-velocity and fluctuation profiles above the cylinder top surface. Overall, profiles of both models agree well with the experiment of Lyn \& Rodi 


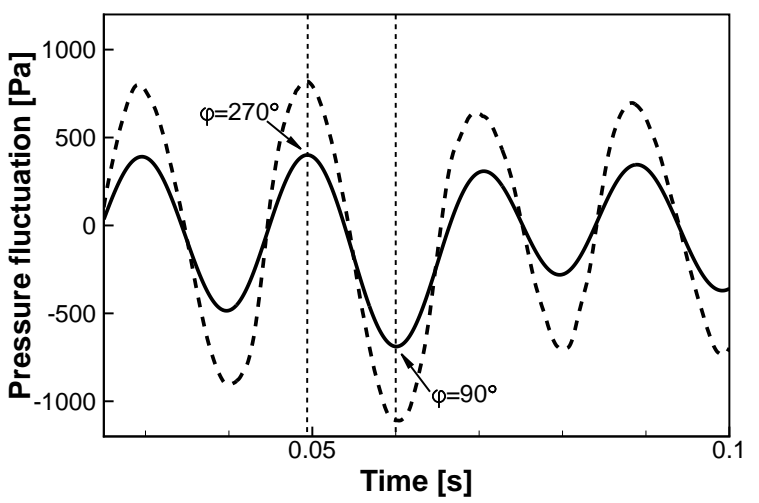

Figure 8: Definition of phase angles on filtered pressure signal: -- , Raw pressure; Filtered pressure

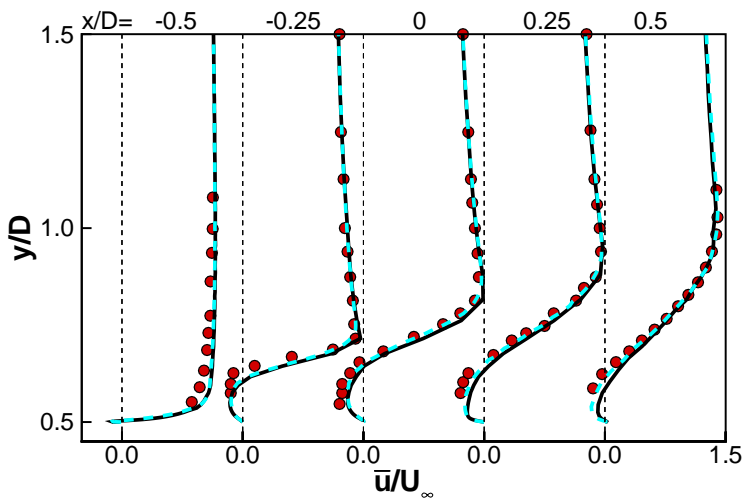

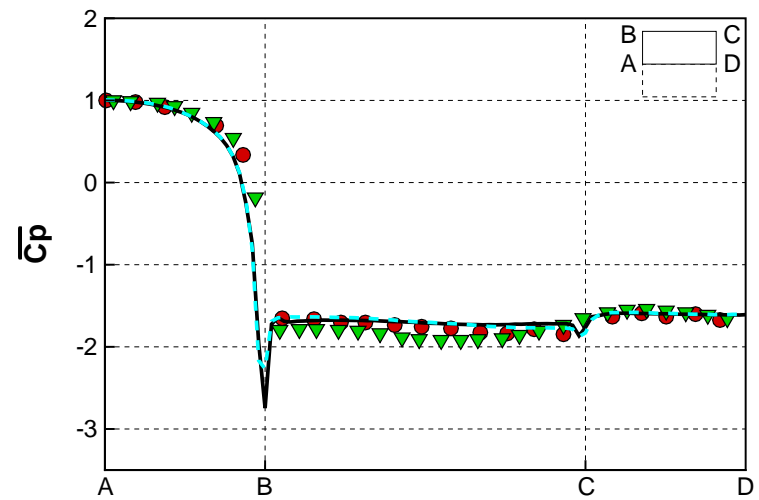

Figure 9: Time-averaged surface pressure coefficient: —, LES-SA; --, LES- $k \omega ; \bullet$ and $\nabla$, Exp.

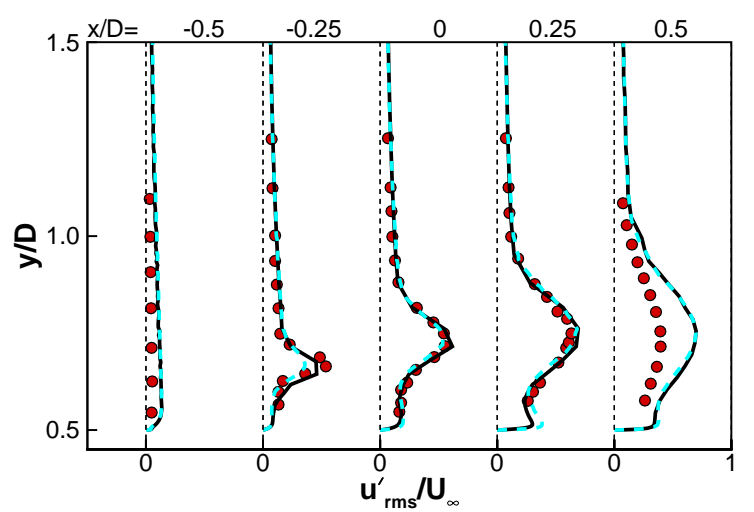

Figure 10: Time-averaged and fluctuate $x$-velocity above the cylinder top surface: - , LES-SA; ,-- LES- $k \omega ; \bullet$, Exp.

$(1994)(\bullet)$. The shear layer position, where the maximum velocity is reached, is accurately captured. A slightly higher velocity of the reversed flow is shown by LES- $k \omega$ model near the rear surface $(x=0.5 D)$. This is believed to be the result of more active vortices in the near wake. Overestimate can be found in the $x$-velocity fluctuation profiles at $x=0.5 \mathrm{D}$. It is probably due to the over-predicted intensity of interaction between the flow recirculation and the shear layer.

Time-averaged streamwise and transverse velocity profiles in the wake are presented in Figure 11, and the fluctuation profiles are shown in Figure 12. Profiles of simulations match with experimental results of Lyn et al. (1995) (•), Durão et al. (1988) (^) and Fohanno \& Martinuzzi (2004) (•). In terms of the $x$-velocity, the LES- $k \omega$ model reports a slightly stronger reversed flow in the near wake $(x \leqslant 1 D)$. As a result, the valley of $x$-velocity profiles is deeper at $x=2 D$ and $x=4.5 D$. This suggests that the LES- $k \omega$ model presents more energetic vortices in the near wake, thus reduces the recovery speed of $x$-velocity. Both models slightly overpredict the $y$-velocity fluctuation at $x=1 \mathrm{D}$ after the cylinder $(-0.5 D \leqslant y \leqslant 0.5 D)$ while under-prediction of the peak occurs at $x \geqslant 2 D$. On the other hand, profiles of the $y$-velocity and $x$-velocity fluctuation are close between the numerical results and the experimental data. Overall, it is obvious that profiles of the LES- $k \omega$ model agree slightly better with that of the experiments.

Figure 13 presents the streamwise velocity recovery and velocity fluctuation at $y=0$ in the 

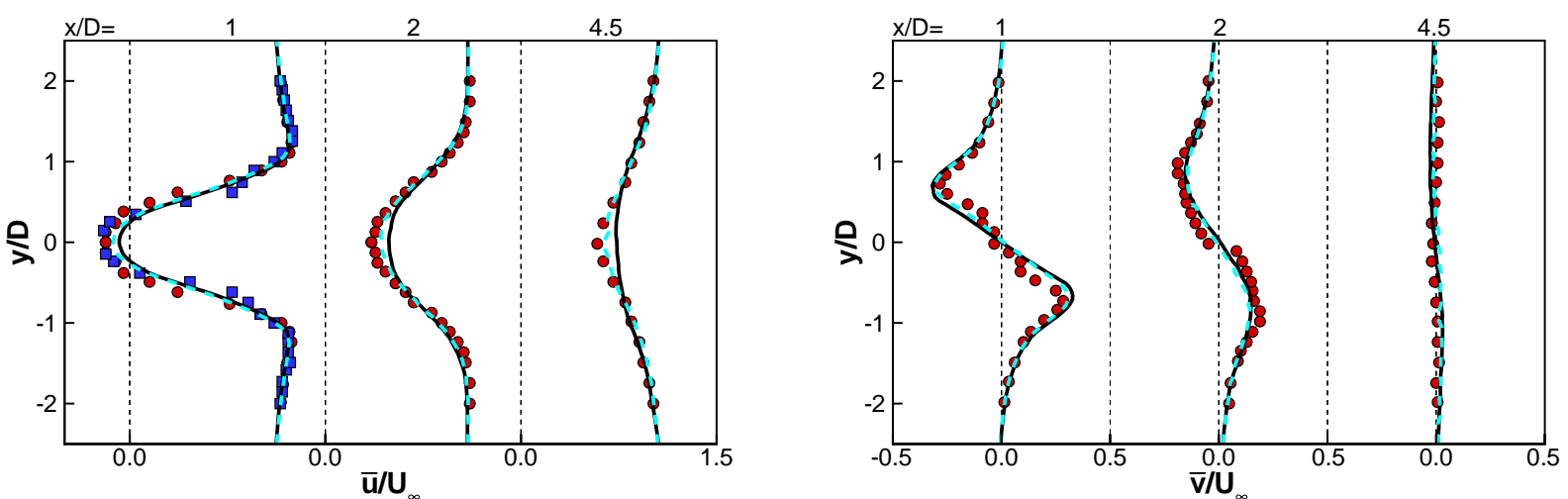

Figure 11: Time-averaged $x$ - and $y$-velocity in the wake: - , LES-SA; - -, LES- $k \omega ; \bullet$ and $\bullet$, Exp.
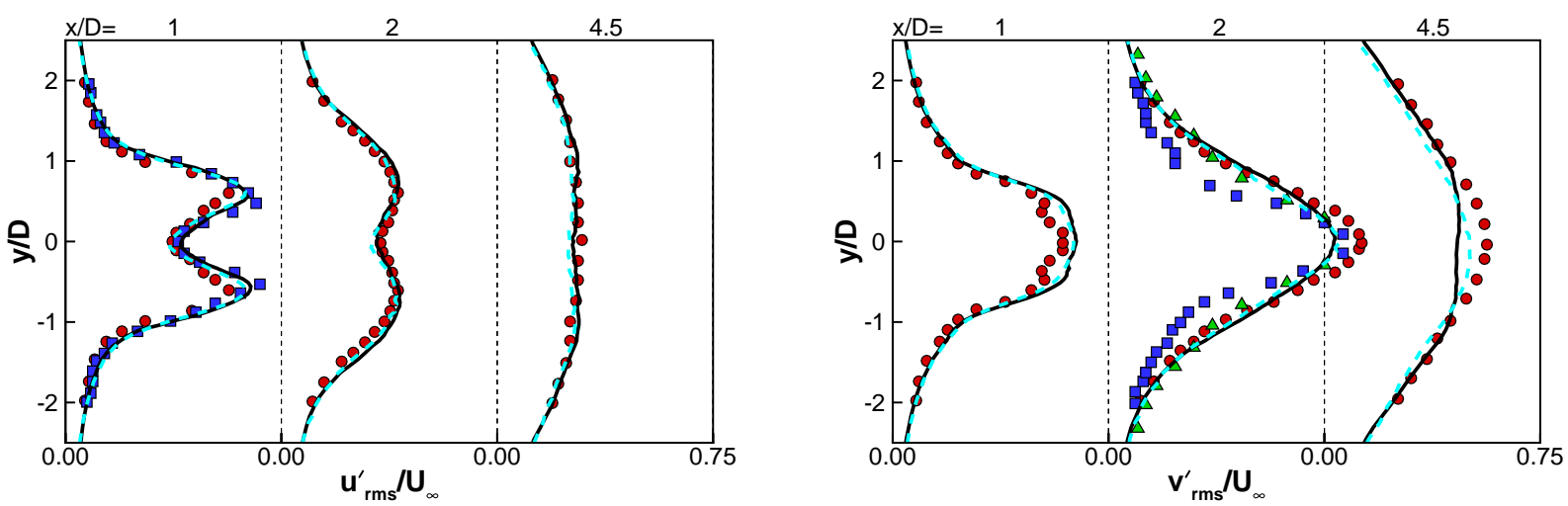

Figure 12: Fluctuate $x$ - and $y$-velocity in the wake:-, LES-SA; --, LES- $k \omega ; \bullet, \wedge$ and $\bullet$, Exp.

wake. The velocity recovery of Durão et al. (1988) ( $\triangle$ ) is faster compared with the other two experiments. Also, the peak of the fluctuation profile is higher and further from the cylinder. This is mainly because that in Durão et al.'s experiment (1988), the Reynolds number is lower $(14,000)$ and the turbulence intensity of the inflow is higher $(6 \%)$. In spite of that, profiles of both models agree with the experimental profiles. However, the LES- $k \omega$ model provides a slightly higher velocity in the reversed flow region $(x \leqslant 1.5 \mathrm{D})$ and relatively slower recovery in the wake, which is the same as the transverse profiles show. As mentioned before, it indicates that the vortices reproduced by the LES- $k \omega$ model have higher backward $x$-velocity and thus leads to a decreased $x$-velocity far from the cylinder. Furthermore, the peaks of the $x$-velocity fluctuation profile of the LES- $k \omega$ model is shifted slightly away from the cylinder, which is closer to that of the experiments. It can be observed from the experimental profile of Lyn et al. (1995) (•) that a small rise in the velocity fluctuation occurs at $3 D \leqslant x \leqslant 4 D$. A similar trend is shown in profiles of Fohanno \& Martinuzzi (2004) (•) and LES-SA model, but not in that of the LES- $k \omega$ model. This is the main reason that the $x$-velocity fluctuation of LES- $k \omega$ at $3 D \leqslant x \leqslant 6 D$ is a little lower than that of the LES-SA and experiments. It may be the vortex shedding phenomenon in the wake that causes this small rise.

\subsection{Phase-averaged results}

Phase-averaged velocities are presented for better understanding of the quasi-periodic flow, which is mainly caused by the von Kármán instability. Figure 7 illustrates the Strouhal number obtained from the FFT of the pressure signal from the cylinder top surface centre. As Table 1 

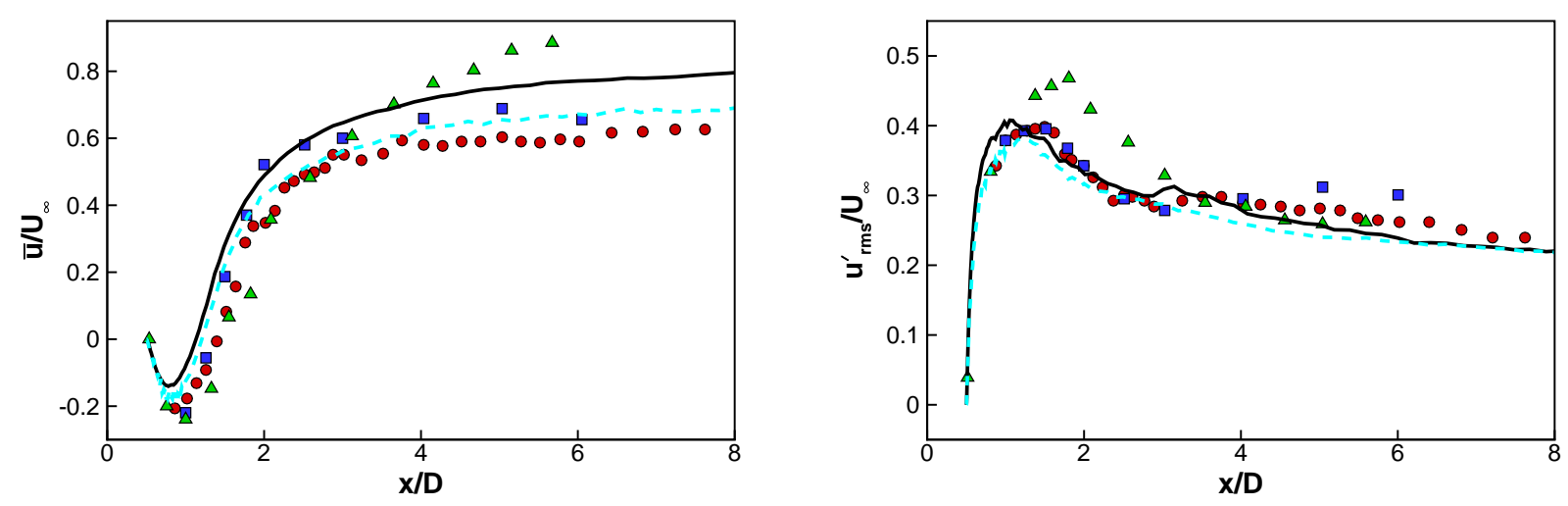

Figure 13: Time-averaged and fluctuate velocity at $y=0$ in the wake: - , LES-SA; - -, LES$k \omega ; \bullet, \Delta$ and $\bullet$, Exp.

Table 1: Comparison of Strouhal number as a reference of vortex shedding frequency

\begin{tabular}{|r|r|r|r|r|r|}
\hline & Lyn \& Rodi & Durão et al. & Bearman \& Obasaju & LES-SA & LES- $k \omega$ \\
\hline$S t$ & 0.134 & 0.133 & 0.130 & 0.134 & 0.130 \\
\hline
\end{tabular}

shows, the Strouhal number of present simulations agree well with relevant experiments.

Phase-averaged $x$-velocity and fluctuation profiles at phase angle $90^{\circ}$ and $270^{\circ}$ are presented in Figure 14. Favourable agreement has been obtained between profiles of LES-SA and experiment of Lyn \& Rodi (1994) (•). The flapping movement of the shear layer is clearly illustrated by profiles at two different phase angles. A very small under-prediction of the phase-averaged $x$-velocity can be found at phase angle $270^{\circ}$ at $x=0.25 \mathrm{D}$ in the reversed flow region. The peak of the $x$-velocity fluctuation of phase angle $270^{\circ}$ at $x=-0.25 D$ is also underestimated. These underestimates reveal that both the strength and the fluctuation of the reversed flow are underestimated, which can be related to the less energetic vortices in the wake that LES-SA demonstrates.

Changes of the velocities in the wake $(x=2 D)$ with phase angles are shown in Figure 15. The phase-averaged velocities above the cylinder $(y \geqslant 1 D)$ are well captured. Underestimate of the amplitude can be found in both the streamwise and transverse velocity profiles after the cylinder $(y \leqslant 0.5 D)$. This indicates that the LES-SA model under-predicts the velocity changes introduced by the vortex shedding in the wake. This under-prediction has proved to have an impact on the reversed flow near the cylinder top/bottom surfaces. Furthermore, the under-predicted reversed flows are expected to reduce the convective heat flux on top/bottom and rear surfaces of the cylinder, thus resulting a lower surface Nusselt.

\subsection{Heat transfer results}

Table 2: Global Nusselt number $\overline{N u}_{g}$ of present simulations and experiments

\begin{tabular}{|l|r|r|r|r|r|}
\hline & Igarashi & Yoo et al. & LES-SA & LES- $k \omega$ & LES-SA (FST) \\
\hline$\overline{N u}_{g}$ & 107.6 & 111.6 & 89.3 & 96.9 & 91.5 \\
\hline
\end{tabular}



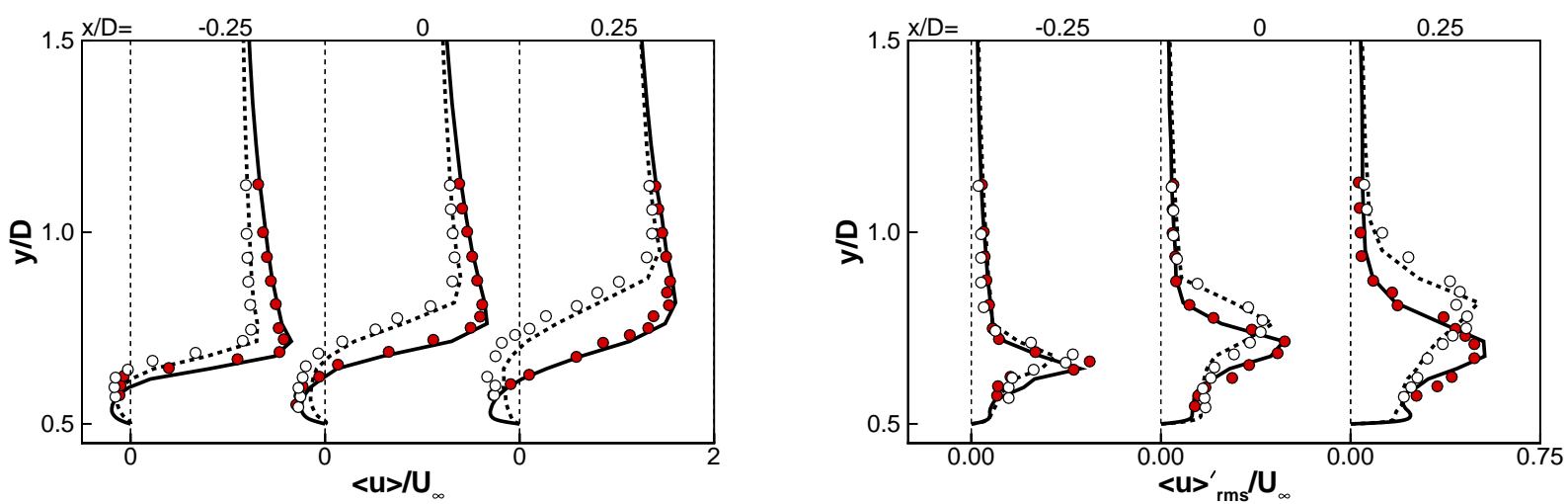

Figure 14: Phase-averaged $x$-velocity above the cylinder top surface at phase angle $90^{\circ}$ and $270^{\circ}$ : - and - -, LES-SA; • and $\circ$, Exp.
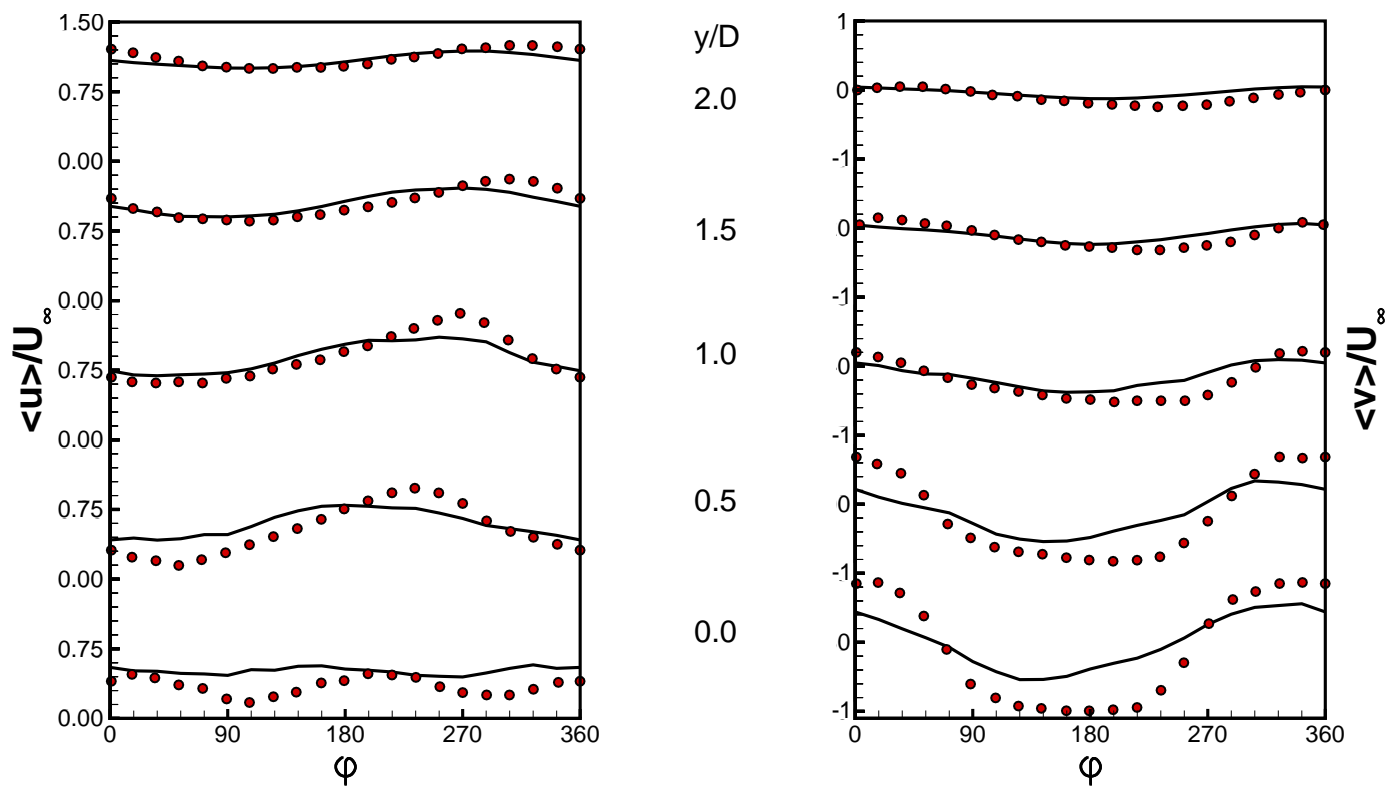

Figure 15: Phase-averaged velocities at $x=2 D$ in the wake: - LES-SA; •, Exp.

A comparison of the time-averaged global Nusselt number on cylinder surfaces are provided in Table 2. Experimental results of Igarashi (1985) $(R e=18,500)$ and Yoo et al. (2003) ( $R e=22,500)$ are scaled to the present Reynolds number by the correlation provided. FST increases the global Nusselt number as expected, while the LES- $k \omega$ model provides a much closer value to the experiments. A more detailed comparison is performed by plotting the timeaveraged local Nusselt number profiles on cylinder surfaces. As Figure 16 shows, profiles of simulations are close to the experiments of Igarashi (1985) and Yoo et al. (2003). They show a good match with the experimental data on the front surface. However, underestimate occurs from approximately half of the top surface to the rear surface probably because of the underpredicted velocity of reversed flow close to the cylinder. A small enhancement in heat transfer on the top surface is found with the imposed FST despite that no obvious difference is found in the velocity profiles. The LES- $k \omega$ model shows better results on the top surface because of the well predicted flow field near the cylinder. No improvement on heat transfer is found with the FST on the rear surface, while LES- $k \omega$ model gives a closer profile to the experiments. This improvement over LES-SA is a result of the better predicted vortex shedding and reversed 


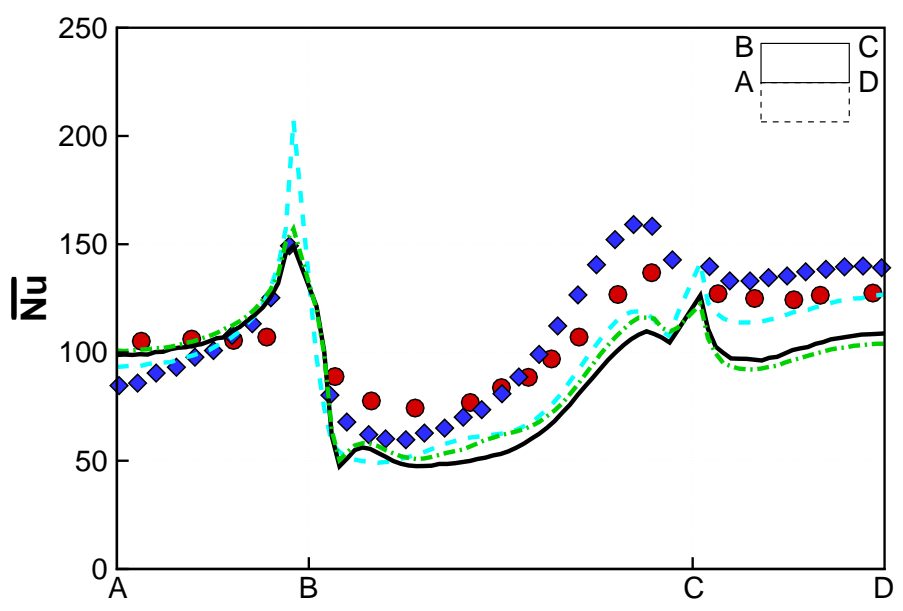

Figure 16: Time-averaged local surface Nusselt number: —, LES-SA; - -, LES- $k \omega ;-\cdot-$, LES-SA (FST); •, Igarashi (1985); •, Yoo et al. (2003)

flows. It may suggest that LES- $k \omega$ is better suited for resolving the unsteady flows near the cylinder.

\section{Conclusions}

Flow passing a heated square cylinder is investigated numerically using hybrid LES-RANS approach. Time-averaged velocities and fluctuations are accurately predicted by both models. The LES- $k \omega$ model reports a slightly stronger reversed flow near the cylinder top/bottom surfaces and in the wake, which agrees more with the experiments. Agreement is obtained between results of the LES-SA model and experiment on the phase-averaged velocity profiles above the top surface and in the wake, despite slight underestimate being found in the wake after the cylinder. In terms of heat transfer, encouraging results of time-averaged global and local Nusselt number are obtained on cylinder surfaces. Small under-prediction is found on top/bottom and rear surfaces due to the difficulties in resolving the flow separation and recirculation close to the cylinder. The imposed free-stream turbulence in the up-stream increases convective heat flux on the top/bottom surface as expected, but no improvement is found on the rear surface. A better prediction is obtained by the LES- $k \omega$ model as a result of more realistically captured reversed flow near the cylinder and vortex shedding in the wake.

\section{References}

1. W.D. Baines and E.G. Peterson. An Investigation of Flow through Screens. Trans. ASME, Vol. 73, pp. 467- 480, 1951.

2. M.F. Barone and C.J. Roy. Evaluation of Detached Eddy Simulation for Turbulent Wake Applications. AIAA J., Vol. 44, No. 12, pp. 3062- 3071, 2006.

3. P.W. Bearman and E.D. Obasaju. An Experimental Study of Pressure Fluctuations on Fixed and Oscillating Square-section Cylinders. J. Fluid Mech., Vol. 119, pp. 297- 321, 1982.

4. M. Boileau, F. Duchaine, J.C. Jouhaud and Y. Sommerer. Large Eddy Simulation of Heat Transfer around a Square Cylinder using Unstructured Grids. AIAA J., Vol. 51, No. 2, pp. 372- 385, 2013.

5. G. Bosch and W. Rodi. Simulation of Vortex Shedding Past a Square Cylinder with Different Turbulence Models. Int. J. Numer. Meth. Fl., Vol. 28, Issue 4, pp. 601- 616, 1998. 
6. G.E.P. Box and M.E. Muller A Note on the Generation of Random Normal Deviates. Ann. Math. Statist., Vol. 29, No. 2, pp. 610- 611, 1958.

7. K. Cho, T.F. Irvine and J. Karni. Measurement of the Diffusion Coefcient of Naphthalene into Air. Int. J. Heat Mass Tran., Vol. 35, No. 4, pp. 957- 966, 1992.

8. D. Durão, M. Heitor and J. Pereira. Measurements of Turbulent and Periodic Flows around a Square Cross-section Cylinder. Exp. Fluids, Vol. 6, Issue 5, pp. 298- 304, 1988.

9. S. Fohanno and R.J. Martinuzzi. A Phase-Averaged Analysis of Droplet Dispersion in the Wake of a Square Cylinder in a Uniform Stream. J. Fluids Eng., Vol. 126, No. 1, pp. 110119, 2004.

10. T. Igarashi. Heat Transfer from a Square Prism to an Air Stream. Int. J. Heat Mass Tran., Vol. 28, Issue 1, pp. 175- 181, 1985.

11. D.A. Lyn and W. Rodi. The Flapping Shear Layer Formed by Flow Separation from the Forward Corner of a Square Cylinder. J. Fluid Mech., Vol. 267, pp. 353- 376, 1994.

12. D.A. Lyn, S. Einav, W. Rodi and J.H. Park. A Laser-Doppler Velocimetry Study of Ensemble-averaged Characteristics of the Turbulent Near Wake of a Square Cylinder. $J$. Fluid Mech., Vol. 304, pp. 285- 319, 1995.

13. F.R. Menter, M. Kuntz and R. Langtry. Ten Years of Industrial Experience with the SST Turbulence Model. In Turbulence, Heat and Mass Transfer 4, ed: K. Hanjalic, Y. Nagano, and M. Tummers, Begell House, Inc., pp. 625- 632, 2003.

14. A.M. Mitchell, S.A. Morton, J.R. Frosythe and R.M. Cummings. Analysis of Delta-Wing Vortical Substructures Using Detached-Eddy Simulation. AIAA J., Vol. 44, No. 5, pp. $964-$ 972, 2006.

15. C.D. Pakora, W.A. McMullan, G.J. Page and J.J. McGuirk. Influence of a numerical boundary layer trips within LES of a subsonic jet on spatio-temporal correlations. In 17th AIAA/CEAS Aeroacoustics Conference (32nd AIAA Aeroacoustics Conference), AIAA 2011-2920, Portland, Oregon, 2011.

16. W. Rodi, J.H. Ferziger, M. Breuer and M. Pourquiée. Status of Large Eddy Simulation: Results of a Workshop. J. Fluids Eng., Vol. 119, No. 2, pp. 248- 262, 1997.

17. S. Wiesche. Large-eddy Simulation Study of an Air Flow past a Heated Square Cylinder. Heat Mass Transfer, Vol. 43, Issue 6, pp. 515- 525, 2007.

18. H. Xia. Dynamic grid detach-eddy simulation for synthetic jet flows. Ph.D. thesis, The University of Sheffield, 2005.

19. H. Xia and P.G. Tucker. Numerical Simulation of Single-Stream Jets from a Serrated Nozzle. Flow Turbulence Combust., Vol. 88, pp. 3- 18, 2012.

20. S.Y. Yoo, J.H. Park, C.H. Chung and M.K. Chung. An Experimental Study on Heat/Mass Transfer From a Rectangular Cylinder. J. Heat Transf., Vol. 125, Issue 6, pp. 1163- 1169, 2003. 